\title{
Pharmacist review and its impact on Singapore nursing homes
}

\author{
Hui Shan Chia ${ }^{1}$, MClinPharm, BSc Pharm (Hons), John Aik Hui $\underline{H o}^{2}$, BPharm, Bernadette Daolin Lim³ ${ }^{3}$, BSc Pharm (Hons)
}

INTRODUCTION There is a high prevalence of polypharmacy and inappropriate medication use in Singapore nursing homes. This study primarily explored the benefits of pharmacist reviews in local nursing homes. The secondary aims were to review the potential cost savings gained from following the pharmacists' recommendations and to identify the possible risks associated with polypharmacy and inappropriate medication use.

METHODS A retrospective period prevalence study was performed. We analysed the pharmacotherapy problems highlighted by pharmacists in three nursing homes and the rate of acceptance of pharmacists' recommendations. Data was collected in two phases: (a) a one-month pre-setup period, during which 480 patients were reviewed (i.e. one-time review before weekly pharmacist visits); and (b) a six-month post-setup period, during which the 480 patients were reviewed again. Pharmacotherapy problems were classified according to a clinical pharmacist recommendation taxonomy and potential risks were identified. Monthly cost savings were calculated and compared with the monthly costs of pharmacist reviews. RESULTS A total of 392 pharmacotherapy problems were identified, with pharmacist recommendations noted for each problem. Among the 392 recommendations, 236 (60.2\%) were accepted. The pharmacotherapy problems were analysed for potential risks, including falls $(16.0 \%)$ and constipation $(13.1 \%)$. The acceptance rates were higher during the postsetup period compared to the pre-setup period $(p<0.0001)$. Total direct acquisition cost savings during the pre- and post-setup periods were SGD 388.30 and SGD 876.69, respectively.

CONCLUSION The provision of pharmaceutical care to nursing home residents resulted in improved medication safety and quality of care.

Keywords: nursing homes, pharmacists, Singapore

\section{INTRODUCTION}

There is a high prevalence of polypharmacy and inappropriate medication use among nursing homes in Singapore. ${ }^{(1)}$ The global phenomenon of inappropriate prescribing and lack of periodic reviews can result in drug-related complications in nursing homes. ${ }^{(2-5)}$ Polypharmacy and inappropriate medication use have been associated with increased costs, drug interactions, adverse effects and hospitalisations. ${ }^{(5)}$ Studies have demonstrated that interventions by pharmacists can promote correct medication use, thus contributing to improvements in the therapeutic outcomes of nursing home residents. ${ }^{(3,4)}$

In an interview study, Halvorsen et $\mathrm{al}^{\left({ }^{(6)}\right.}$ examined the effects of a multidisciplinary collaboration between nursing homes physicians/nurses and pharmacists, as well as the impact and structure of such a collaboration. The study revealed that physicians and nurses valued the services rendered by pharmacists. It also reported an improvement in the residents' drug therapy as a result of the collaboration, although it concluded that a more structured framework was required to support such collaborations.

Forsetlund et $\mathrm{al}^{\left({ }^{(4)}\right.}$ reviewed interventional studies aimed at partially or completely reducing potentially inappropriate use or prescription of medication among elderly nursing home residents, as well as studies that measured drug use. It was reported that, under certain circumstances, education and pharmacist medication reviews helped to reduce inappropriate drug use; however, the authors found that the evidence in the reviewed studies was of low quality.

In its 2004 Guidelines on Medication Management in Nursing Homes, the Ministry of Health, Singapore, recommended the provision of pharmaceutical care by pharmacists to nursing home residents. ${ }^{(7)}$ An increasing number of local nursing homes have pursued this recommendation. It has been suggested that a multidisciplinary approach involving geriatricians, nursing home physicians, nurses and pharmacists could potentially address polypharmacy and the inappropriate use of medication. ${ }^{(1)}$ As pharmacists are a relatively new addition to the multidisciplinary team in nursing homes, physicians and nurses may face some initial challenges in this new collaboration; this may especially be the case for physicians, who may feel that their prescribing practices are being unfairly questioned. ${ }^{(8)}$

In Singapore, the role of the pharmacist in nursing homes has seen a gradual shift toward greater recognition and acceptance by the multidisciplinary team over the years. For example, in one of the nursing homes serviced by the National Healthcare Group (NHG) Pharmacy, pharmacists have been participating in regular case conferences involving the multidisciplinary team since December 2009. Prior to a case conference, the pharmacist reviews each patient's medications and existing medical care plan to formulate a subsequent care plan. During the case conference, all medical, nursing and allied health professionals present their individual plans for each resident. These plans are then compiled

${ }^{1}$ Jurong Polyclinic, ${ }^{2}$ Choa Chu Kang Polyclinic, ${ }^{3}$ Pharmacy Services Centre, National Healthcare Group, Singapore

Correspondence: Ms Chia Hui Shan, Senior Pharmacist, National Healthcare Group - Jurong Polyclinic, 3 Fusionopolis Link, \#03-08, Singapore 138543. chia.hui.shan@gmail.com 
to form a holistic general plan for the resident. The pharmacist may also provide suggestions for any problems faced by the other team members. To the best of our knowledge, this is currently the only nursing home in Singapore that involves pharmacists in multidisciplinary case conferences, although plans are in place to implement such collaborations in other nursing homes.

The present study's primary aim was to explore the benefits of pharmacist medication reviews in Singapore nursing homes. This was done through the analysis of the pharmacotherapy problems highlighted by pharmacists and the rate of acceptance of these recommendations by the medical team. The secondary aims of the study were to review the cost savings gained from pharmacist reviews, if any, and to identify the possible risks associated with polypharmacy and the inappropriate use of medication.

\section{METHODS}

This was a retrospective period prevalence study. Data was collected in two distinct phases: (a) a one-month pre-setup period, during which all the enrolled patients were reviewed; a one-time pharmacist review was conducted prior to the weekly pharmacist visits (where the pharmacist spent 5-15 minutes on each patient); and (b) a six-month post-setup period, during which all the patients were reviewed again. Each weekly pharmacist visit lasted for about two hours and 10-15 patients were reviewed at each visit. This study was approved by the Domain Specific Review Board of NHG, Singapore.

For each of the nursing homes included in the present study, two to three pharmacists were involved in the reviews during the pre-setup period, and each pharmacist worked individually. In the post-setup period, only one pharmacist was involved in the review at each nursing home. A total of 480 residents from three nursing homes $(\mathrm{NH} 1, \mathrm{NH} 2$ and $\mathrm{NH} 3)$ were involved in this study. The nursing homes were serviced by pharmacists from the NHG Pharmacy. Details of the population of nursing home residents are shown in Fig. 1, which shows the details of the residents in the three nursing homes and the phases of data collection.

During the review, the pharmacists looked through the inpatient medication records, case notes and relevant medical charts. When required, they observed or interviewed the residents, or obtained a better understanding of the residents' conditions from the nurses. In the pre-setup period, the pharmacists wrote detailed reviews to summarise the pharmacotherapy problems. These reviews were then handed to the doctors for their perusal. In the post-setup period, the pharmacists approached the doctors in a variety of ways (e.g. direct verbal interactions with the doctors, making annotations in the case notes and/or medication records to alert the doctors), depending on the preference of the medical team at each nursing home.

Pharmacotherapy problems highlighted by the pharmacists were recorded in an intervention database belonging to the NHG Pharmacy Services Centre and documented in the case notes of the nursing homes. The problems were then classified according to the clinical pharmacist recommendation (CPR) taxonomy developed by Hoth et al..$^{(9)}$

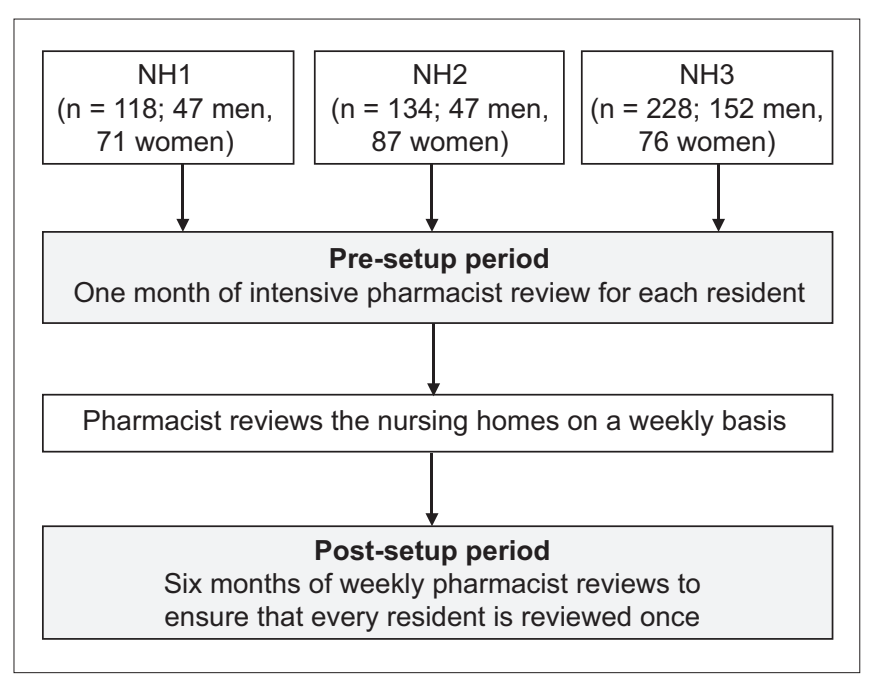

Fig. 1 Flowchart shows details of residents and phases of data collection in the three nursing homes $(\mathrm{NH} 1-3)$.

One of the authors was responsible for classifying the identified pharmacotherapy problems according to the aforementioned CPR taxonomy. The resulting classifications were checked by two other co-authors. The pharmacotherapy problems were also classified according to potential risks such as drug allergy, falls, constipation and abdominal discomfort. These included actual risks that were experienced by the resident but not flagged by the nursing home staff. For example, although low blood glucose $(<4 \mathrm{mmol} / \mathrm{L})$ is indicative of hypoglycaemia and not just potential risk, such cases were classified as potential risks for the purpose of consolidation due to the small number of such cases. Drug references from Lexicomp ${ }^{\circledR}$ and Micromedex ${ }^{\circledR}$ Healthcare Series were consulted to formulate the list of potential risks identified. Each pharmacotherapy problem could be classified with more than one potential risk.

The costs of drugs were calculated in Singapore dollars using non-subsidised, private rates from iPharm, a software program used by NHG Pharmacy. The monthly cost saving was defined as the monthly costs of drugs discontinued or substituted as a result of the pharmacist review. The monthly cost saving was compared with the monthly cost of pharmacist reviews to compute the nett savings.

In the post-setup period, each pharmacist review with a resident took approximately 5-15 minutes. Based on a rate of SGD 60 per hour, a five-minute review of 80 residents would cost SGD 400 per month, while a 15-minute review would cost SGD 1,200 per month. The cost of the pharmacist reviews averaged out to be about SGD 800 per month for 80 patients (calculated based on the average of SGD 400 and SGD 1,200 per month). For the pre-setup period, the cost of the pharmacist reviews was multiplied by six (i.e. SGD 4,800), as all 480 patients were reviewed in a single month.

Statistical analysis was performed using GraphPad QuickCalcs (GraphPad Software Inc, La Jolla, CA, USA). Fisher's exact test was used to examine the proportion of pharmacist recommendations that were accepted during the post-setup period as compared 
to the pre-setup period. All analyses were two-tailed and a $\mathrm{p}$-value $<0.05$ indicated significance.

\section{RESULTS}

A total of 392 pharmacotherapy problems with recommendations were reported during the pre- and post-setup periods. Among these, $236(60.2 \%)$ were accepted. The acceptance rates during the pre-setup period and post-setup period are shown in Table I. There was an increase in the cumulative acceptance rate across the three nursing homes from the pre- to the postsetup period $(p<0.0001)$. However, when the three nursing homes were compared separately, only $\mathrm{NH} 1$ showed a statistically significant improvement in the rate of acceptance $(\mathrm{p}<0.0001)$.

We analysed the proportion of residents with varying numbers of pharmacotherapy problems (Table II). In the pre-setup period, each resident had approximately 0.429 pharmacotherapy problems, whereas in the post-setup period, each resident had approximately 0.388 pharmacotherapy problems. We also examined the types of pharmacotherapy problems identified and ranked these problems against the rate of acceptance (Tables III \& IV). In the pre-setup period, 'Compliance or drug administration issue' had the highest acceptance rate (i.e. $100 \%$ ), although there were only two counts of that problem. 'Inappropriate/suboptimal dose', which had the highest number of acceptance counts, signified a more prevalent problem with a greater impact. In the post-setup period, the most prevalent problem (i.e. 44 counts) with the highest acceptance rate (i.e. $95.5 \%$ ) was 'Patient no longer taking medicine on profile or needs a new prescription for medicine not on profile'. The problem 'Medication use without indication or unclear indication' had the second highest prevalence, with an acceptance rate of $72.7 \%$.

The pharmacotherapy problems identified by the pharmacists were classified according to the taxonomy developed by Hoth et $\mathrm{al}^{(9)}$ (Table V). The most prevalent pharmacotherapy problems were as follows: 'Inappropriate/suboptimal dose' (16.8\%); 'Medication use without indication or unclear indication' $(16.3 \%)$; 'Patient no longer taking medicine on profile or needs a new prescription for medicine not on profile' $(12.0 \%)$; and 'Untreated condition' (11.7\%).

The pharmacotherapy problems identified were analysed for potential risks (Table VI). A total of 375 potential risks were found - 177 and 198 from the pre- and post-setup periods, respectively. The most common risks were falls $(16.0 \%)$ and constipation (13.1\%), which were mostly caused by the prolonged use of antihistamines; the pharmacists subsequently recommended that the medication be discontinued. Other important risks identified included drug allergy (0.5\%), hyperkalaemia $(1.3 \%)$, hypoglycaemia $(1.6 \%)$, poor seizure control or anti-epileptic toxicity (2.9\%), and drug toxicity due to lack of renal adjustment (2.7\%). One drug could be associated with more than one risk. For example, an anticholinergic drug was included in the risk categories of 'Constipation' and 'Retention of urine'.
Table I. Pharmacotherapy problems/recommendations and acceptance rates of recommendations in the three nursing homes (NH1-3).

\begin{tabular}{lccc}
\hline $\begin{array}{l}\text { Study } \\
\text { phase }\end{array}$ & $\begin{array}{c}\text { No. of problems/ } \\
\text { recommendations }\end{array}$ & $\begin{array}{c}\text { No. of accepted } \\
\text { recommendations }\end{array}$ & $\begin{array}{c}\text { Acceptance } \\
\text { rate (\%) }\end{array}$ \\
\hline NH1 & & & \\
Pre-setup & 72 & 26 & 36.1 \\
$\begin{array}{l}\text { Post-setup } \\
\text { NH2 }\end{array}$ & 104 & 73 & $70.2^{*}$ \\
Pre-setup & 69 & 51 & 73.9 \\
Post-setup & 41 & 35 & $85.4^{+}$ \\
NH3 & & & \\
Pre-setup & 65 & 28 & 43.1 \\
Post-setup & 41 & 23 & $56.1^{*}$ \\
Combined" & & & \\
Pre-setup & 206 & 105 & 51.0 \\
Post-setup & 186 & 131 & $70.4^{*}$ \\
Overall & 392 & 236 & 60.2 \\
\hline
\end{tabular}

$\star_{p}<0.0001 . t_{p}=0.2325 .{ }_{p}=0.2328$. ๆ Combined values for all three nursing homes.

Table VII shows selected details on patients identified with potentially serious risks, namely hypoglycaemia, falls, prolonged nonsteroidal anti-inflammatory drugs (NSAIDs) exposure, poor seizure control or antiepileptic toxicity, and drug toxicity due to lack of renal adjustment. These risks are known to be deleterious but not uncommon among the elderly.

The recommendations made by the pharmacists to resolve the pharmacotherapy problems were categorised according to whether the recommendations provided direct acquisition cost savings (through the discontinuation of drugs that were no longer required). Total direct cost savings (calculated based on the one month cost of discontinued drugs) during the pre-setup period were SGD 388.30, while those during the post-setup period were SGD 876.69. The mean cost saving per recommendation was SGD 12.94 during the pre-setup period and SGD 19.06 during the post-setup period. However, after the costs of the intensive pharmacist reviews (conducted during the pre-setup period) were factored in, the direct cost savings for the pre-setup period did not cover the cost of pharmacist review (nett saving of -SGD 4,411.70); during the post-setup period, nett saving of SGD 76.69 per month was achieved.

The monthly direct cost savings resulting from the pharmacists' recommendations were further analysed for each nursing home. For $\mathrm{NH} 1$, there were seven identified problems that could potentially save costs in the pre-setup period (SGD 63.41 of potential monthly direct cost savings). In the post-setup period, 26 problems that had the potential for cost savings were identified (SGD 306.69 of potential direct monthly cost savings). For $\mathrm{NH} 2$, there were 17 identified problems in the pre-setup period, resulting in SGD 104.98 of potential direct monthly cost savings; in the post-setup period, there were 13 identified problems, resulting in SGD 168.13 of potential direct monthly cost savings. For $\mathrm{NH} 3$, seven problems each were identified in the pre- and post-setup periods, resulting in SGD 219.91 and SGD 401.87 of potential monthly direct cost savings, respectively. 
Table II. Proportion of nursing home (NH) residents with varying numbers of pharmacotherapy problems.

\begin{tabular}{|c|c|c|c|c|c|c|}
\hline \multirow{2}{*}{$\begin{array}{l}\text { No. of pharmacotherapy } \\
\text { problems }\end{array}$} & \multicolumn{2}{|c|}{$\mathrm{NH} 1(\mathrm{n}=118)$} & \multicolumn{2}{|c|}{ NH2 $(n=134)$} & \multicolumn{2}{|c|}{ NH3 $(n=228)$} \\
\hline & Pre-setup & Post-setup & Pre-setup & Post-setup & Pre-setup & Post-setup \\
\hline 0 & $66(55.9)$ & $56(47.5)$ & $88(65.7)$ & $68(50.7)$ & $178(78.1)$ & $178(78.1)$ \\
\hline 1 & $35(29.7)$ & 39 (33.1) & $27(20.1)$ & $52(38.8)$ & $31(13.6)$ & $30(13.2)$ \\
\hline 2 & $12(10.2)$ & 11 (9.3) & $13(9.7)$ & $9(6.7)$ & $18(7.9)$ & $4(1.8)$ \\
\hline 3 & $5(4.2)$ & $7(5.9)$ & $6(4.5)$ & $5(3.7)$ & $1(0.4)$ & $1(0.4)$ \\
\hline 4 & $0(0)$ & $3(2.5)$ & $0(0)$ & $0(0)$ & $0(0)$ & $0(0)$ \\
\hline 5 & $0(0)$ & $2(1.7)$ & $0(0)$ & $0(0)$ & $0(0)$ & $0(0)$ \\
\hline
\end{tabular}

Table III. Types of pharmacotherapy problems identified during the pre-setup period, ranked according to their acceptance rates.

\begin{tabular}{lccc}
\hline Type of intervention & $\begin{array}{c}\text { No. of problems/ } \\
\text { recommendations }\end{array}$ & $\begin{array}{c}\text { No. of accepted } \\
\text { recommendations }\end{array}$ & $\begin{array}{c}\text { Acceptance } \\
\text { rate (\%) }\end{array}$ \\
\hline Compliance or drug administration issue & 2 & 2 & 100.0 \\
Patient no longer taking medicine on profile or needs a & 3 & 5 & 66.7 \\
new prescription for medicine not on profile & 8 & 10 & 62.5 \\
Undertreated condition & 16 & 21 & 62.5 \\
Inappropriate/suboptimal/inconvenient schedule & 34 & 6 & 61.8 \\
Untreated condition & 5 & 8 & 60.0 \\
Inappropriate/suboptimal route & 11 & 24 & 54.5 \\
Therapeutic monitoring for toxicity & 15 & 2 & 53.3 \\
Potential ADE/ADR & 46 & 9 & 52.2 \\
Inappropriate/suboptimal dose & 4 & 1 & 50.0 \\
Actual ADE/ADR & 18 & 2 & 50.0 \\
Alternative therapy & 2 & 2 & 50.0 \\
Minimal/no evidence of therapeutic effectiveness & 4 & 8 & 50.0 \\
Therapeutic duplication & 6 & 0 \\
Therapeutic monitoring for effectiveness & 31 & 1 & 28.6 \\
Medication use without indication or unclear indication & & 25.8 \\
Less expensive, equally effective alternative & & 0 \\
\hline
\end{tabular}

ADE: adverse drug effect; ADR: adverse drug reaction

Table IV. Types of pharmacotherapy problems identified during the post-setup period, ranked according to their acceptance rates.

\begin{tabular}{|c|c|c|c|}
\hline Type of intervention & $\begin{array}{l}\text { No. of problems/ } \\
\text { recommendations }\end{array}$ & $\begin{array}{l}\text { No. of accepted } \\
\text { recommendations }\end{array}$ & $\begin{array}{l}\text { Acceptance } \\
\text { rate }(\%)\end{array}$ \\
\hline $\begin{array}{l}\text { Patient no longer taking medicine on profile or needs a } \\
\text { new prescription for medicine not on profile }\end{array}$ & 44 & 42 & 95.5 \\
\hline Compliance or drug administration issue & 9 & 8 & 88.9 \\
\hline Inappropriate/suboptimal/inconvenient schedule & 9 & 8 & 88.9 \\
\hline Therapeutic monitoring for toxicity & 9 & 7 & 77.8 \\
\hline Therapeutic monitoring for effectiveness & 12 & 9 & 75.0 \\
\hline Medication use without indication or unclear indication & 33 & 24 & 72.7 \\
\hline Actual ADE/ADR & 3 & 2 & 66.7 \\
\hline Alternative therapy & 3 & 2 & 66.7 \\
\hline Undertreated condition & 5 & 3 & 60.0 \\
\hline Inappropriate/suboptimal dose & 20 & 12 & 60.0 \\
\hline Inappropriate/suboptimal route & 2 & 1 & 50.0 \\
\hline Less expensive, equally effective alternative & 2 & 1 & 50.0 \\
\hline Therapeutic duplication & 5 & 2 & 40.0 \\
\hline Untreated condition & 12 & 4 & 33.3 \\
\hline Potential ADE/ADR & 16 & 5 & 31.3 \\
\hline
\end{tabular}

$A D E$ : adverse drug effect; ADR: adverse drug reaction 
Table V. Number of pharmacotherapy problems identified during the pre- and post-setup periods, classified according to the taxonomy developed by Hoth et al. ${ }^{(9)}$

\begin{tabular}{lccc}
\hline Type of pharmacotherapy problems & \multicolumn{3}{c}{ No. of pharmacotherapy problems } \\
\cline { 2 - 4 } & Pre-setup ( $\mathbf{n = 2 0 6 )}$ & Post-setup ( $\mathbf{n = 1 8 6 )}$ & Total (\%) (n = 392) \\
\hline Actual ADE/ADR & 4 & 3 & $7(1.8)$ \\
Potential ADE/ADR & 15 & 16 & $31(7.9)$ \\
Therapeutic monitoring for toxicity & 11 & 9 & $20(5.1)$ \\
Medication use without indication or unclear indication & 31 & 33 & $64(16.3)$ \\
Untreated condition & 34 & 12 & $46(11.7)$ \\
Undertreated condition & 8 & 5 & $13(3.3)$ \\
Alternative therapy & 18 & 3 & $21(5.4)$ \\
Minimal/no evidence of therapeutic effectiveness & 2 & 2 & $4(1.0)$ \\
Therapeutic monitoring for effectiveness & 6 & 12 & $18(4.6)$ \\
Compliance or drug administration issue & 2 & 9 & $11(2.8)$ \\
Inappropriate/suboptimal dose & 46 & 20 & $66(16.8)$ \\
Inappropriate/suboptimal/inconvenient schedule & 16 & 9 & $25(6.4)$ \\
Inappropriate/suboptimal route & 5 & 2 & $7(1.8)$ \\
Therapeutic duplication & 4 & 5 & $9(2.3)$ \\
Less expensive, equally effective alternative & 1 & 2 & $3(0.8)$ \\
Patient no longer taking medicine on profile or needs a & 3 & 44 & $47(12.0)$ \\
new prescription for medicine not on profile & & 2 & \\
\hline
\end{tabular}

ADE: adverse drug effect; ADR: adverse drug reaction

Table VI. Potential risks associated with the pharmacotherapy problems identified.

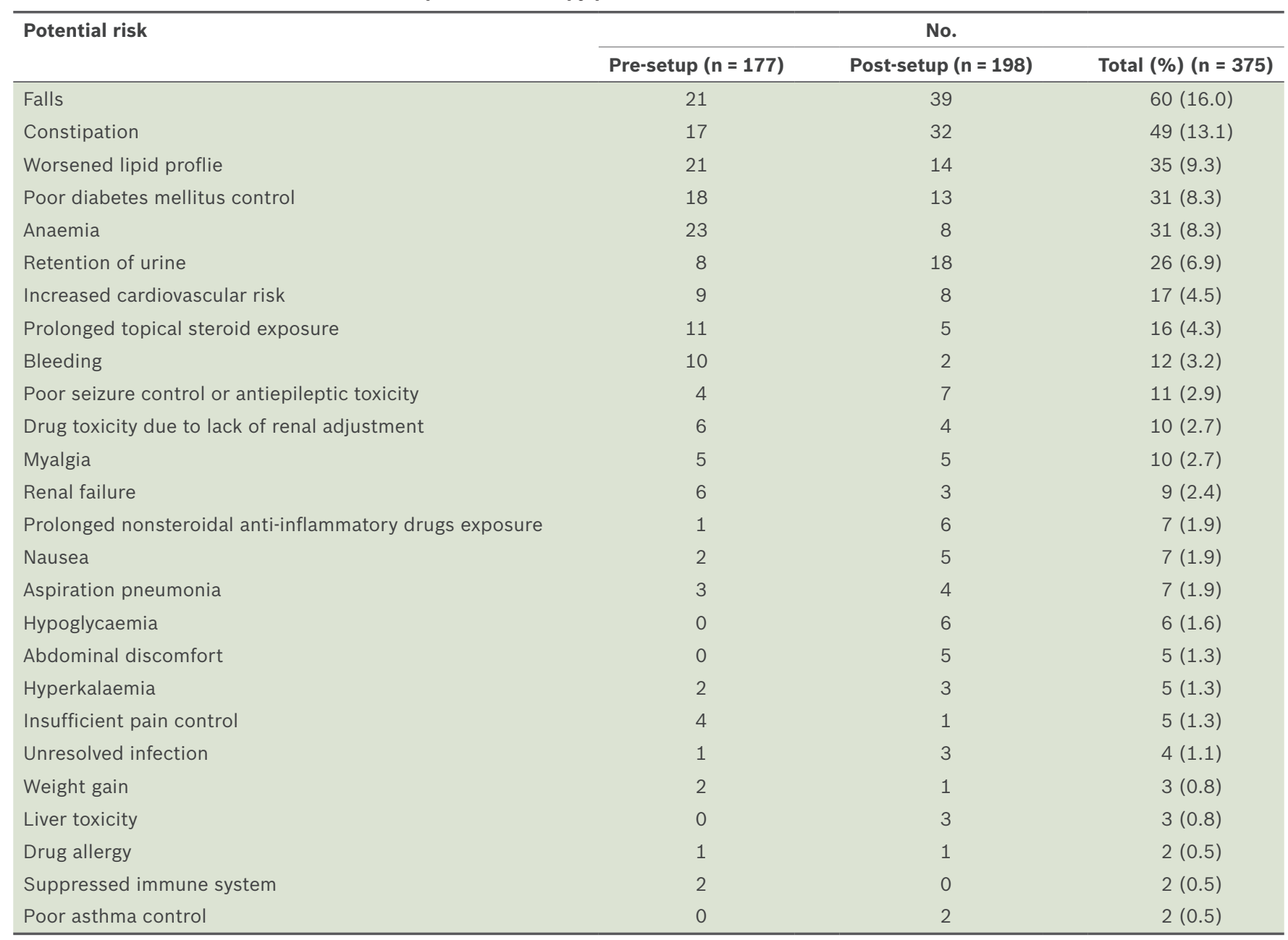


Table VII. Selected details on patients identified with potentially serious risks due to pharmacotherapy problems.

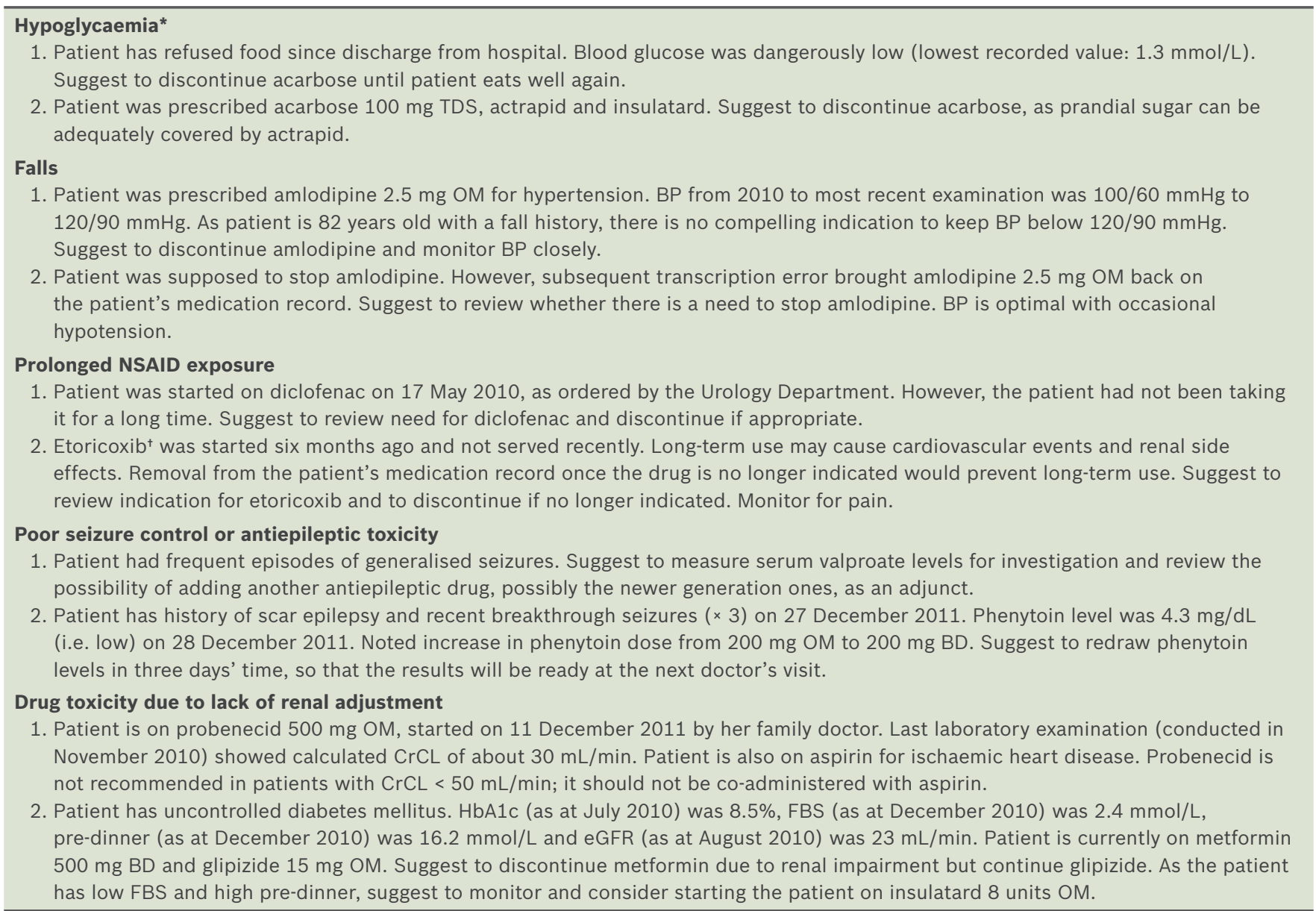

* Note that if a hypoglycaemic event was assessed to be prevented in a patient, this was also recorded as potentially preventing a fall. ${ }^{\dagger}$ Although etoricoxib is not strictly an NSAID, its risk profile is similar; thus, it is categorised as an NSAID for conciseness of classification. BD: twice daily; BP: blood pressure; CrCL: creatinine clearance; eGFR: estimated glomerular filtration rate; FBS: fasting blood glucose; HbA1c: glycated haemoglobin; NSAIDs: nonsteroidal anti-inflammatory drugs; OM: every morning; TDS: three times a day

\section{DISCUSSION}

The present study demonstrated the benefits of pharmacist reviews of prescription orders in Singapore nursing homes. The pharmacists highlighted actual or potential pharmacotherapy problems, quantifying and classifying them accordingly, and also provided recommendations to resolve these problems. From a pharmacoeconomic perspective, there was observable acquisition cost saving derived from such pharmacist reviews during the postsetup period. Most importantly, pharmacist reviews also helped to identify risks, thereby indirectly improving the quality of life and care of nursing home residents, and possibly even reducing morbidity and mortality among elderly residents.

Models of pharmacists' interventions conducted in overseas nursing homes mainly focused on multidisciplinary case conferences, which have proven to be beneficial. ${ }^{(3,8)}$ A meta-analysis showed that pharmacists' interventions using educational outreach, on-site education given alone or as part of an intervention package, and pharmacist medication review may reduce inappropriate drug use; however, the evidence was concluded to be of low quality. ${ }^{(4)}$ In contrast, a quasi-experimental longitudinal study, which evaluated reviews of nationally mandated drug use (not limited to case conferences), found that the effectiveness of medication reviews in improving patient safety in nursing homes was unclear. ${ }^{(10)}$ To the best of our knowledge, the present study is the first in Singapore to address the impact and role of pharmacists' reviews in nursing homes.

The elderly, especially those who are institutionalised, are susceptible to pharmacotherapy problems and risks due to inappropriate medication use. The prevalence of potentially inappropriate drug use (PIDU) ${ }^{(11)}$ has been assessed in elderly hospitalised patients using the Beers criteria ${ }^{(12)}$ and the Screening Tool of Older Person's Prescriptions (STOPP) criteria. ${ }^{(13)}$ In Tosato et al's study, ${ }^{(11)}$ the prevalence of PIDU was $58.4 \%$ when the Beers criteria was applied, $50.4 \%$ when the STOPP criteria was applied and $75.0 \%$ when both sets of criteria were combined, indicating that PIDU is common among elderly hospitalised patients. In another study conducted by Vieira de Lima et al, ${ }^{(14)}$ the Beers criteria was used to assess the prevalence of potentially inappropriate medications (PIM) and the associated factors in a Brazilian elderly population from an institutional setting. ${ }^{(14)}$ The authors showed that there was a high prevalence of PIM in institutionalised elderly Brazilians. ${ }^{(14)}$ In the present study, the Beers criteria was also used by the pharmacists to identify pharmacotherapy problems and to make recommendations, although this was not explicitly mandated in the review process. 
Drug-related problems (DRP) are traditionally classified using a system developed by Hepler and Strand. ${ }^{(15)}$ However, in the present study, we felt that the DRP classification might be overly simplistic and might not cover all the pharmacists' recommendations. Instead, we adopted the CPR taxonomy developed and published by Hoth et al in 2007, as the system allowed for improved description and classification criteria, ${ }^{(9)}$ and was also considered a reliable taxonomy for the clinical pharmacists' recommendations during the time of writing. The CPR taxonomy was developed by clinical pharmacists from the Veterans Affairs Enhanced Pharmacy Outpatient Clinic trial, who performed comprehensive drug therapy reviews and listed specific recommendations. The list of recommendations was then reviewed by an intervention physician, who interviewed the patient and refined the pharmacist's recommendations as needed. Finally, the instrument was refined to reach an acceptable level of reliability. ${ }^{(9)}$

Hatah et $\mathrm{a}^{\left({ }^{(16)}\right.}$ published a paper that explored the interventions performed during adherence support medication reviews, using the DRP Classification Scheme version 6.2 by the Pharmaceutical Care Network Europe (PCNE). ${ }^{(17)}$ The authors found limitations in the use of the PCNE classification for DRP, as the classification did not include all DRPs; they also encouraged future studies to compare the use of DRP classification tools in different types of medication reviews. Although the PCNE classification and CPR taxonomy are relatively similar, we chose the latter, as it is more concise and also enabled us to better classify the pharmacotherapy problems.

Pharmacists are generally expected to be able to identify medication errors and provide education on the appropriate use of medications. ${ }^{(18,19)}$ Thus, the identification of pharmacotherapy problems is one of the major roles of pharmacists at nursing homes. In the present study, 'inappropriate/suboptimal dose' (16.8\%) was identified by our pharmacists as the most prevalent pharmacotherapy problem. This included low blood pressure due to unsuitable doses of hypertensive medicines, lack of dose reduction of proton-pump inhibitors to a maintenance dose, and wrong dose prescribed due to pure human error.

Due to their extensive pharmacotherapeutic training, pharmacists are also able to recommend appropriate therapeutic changes ${ }^{(5)}$ after identifying pharmacotherapy problems. As the majority of nursing home residents require multiple medications, many are at an increased risk of adverse drug effects, drug interactions and hospitalisation. ${ }^{(5)}$ In the present study, our pharmacists identified 'medicine use without indication or unclear indication' (16.3\%) as the second most prevalent problem. To better manage polypharmacy and drug interactions, pharmacists can recommend the discontinuation of medications that lack appropriate indications. As they are more familiar with drug costs and cheaper alternatives within the formulary, pharmacists can also recommend 'less expensive, equally effective alternatives' to help patients and families save costs. Although this constitutes only about $1 \%$ of the recommendations in our study and may seem relatively insignificant, the cost savings can be substantial if the original drug is costly (e.g. switching from a branded product to a generic alternative).
The rate of acceptance of the pharmacists' recommendations was higher in the post-setup period than the pre-setup period $(p<0.0001)$. However, when the acceptance rates were analysed across each nursing home, only $\mathrm{NH} 1$ demonstrated a statistically significant improvement in the acceptance rates, which influenced the overall result. Whether the overall result was due to greater acceptance of the pharmacists' recommendations in $\mathrm{NH} 1$ during the post-setup period or reflected a higher acceptance rate during the pre-setup period in $\mathrm{NH} 2$ and $\mathrm{NH} 3$ is arguable. However, we postulate that the weekly pharmacist visits during the post-setup period (as compared to the once-off visit during the pre-setup period) could have helped the pharmacist build rapport with the nursing home's multidisciplinary team, boosting the acceptance rates. Many studies have examined the collaboration between physicians and pharmacists to promote the safe use of medications. ${ }^{(20,21)}$ Bradley et al highlighted trust, communication, professional respect and 'knowing' each other as the major components of collaboration. ${ }^{(20)}$ In the present study, it is likely that the post-setup period demonstrated a more successful model of pharmacist review because the pharmacists' weekly visits may have improved interdisciplinary communication and trust.

While the direct acquisition cost savings during the pre-setup period did not cover the cost of the pharmacist review (nett saving of -SGD 4,411.70), there was a nett saving of SGD 76.69 per month during the post-setup period. The difference in cost savings is likely due to the rigorous and manpower-intensive nature of the pre-setup pharmacist reviews (i.e. conducted over a short onemonth period) versus the less rigorous and manpower-intensive post-setup reviews (i.e. resources were distributed over six months). However, we recognise the importance of an intensive pre-setup period to eliminate longstanding pharmacotherapy problems before a new pharmacist service is established.

Nonetheless, the present study showed that pharmacist reviews resulted in cost savings for patients. The mean cost savings per recommendation was SGD 12.94 during the pre-setup period and higher, at SGD 19.06, during the post-setup period $(p=0.39)$. Even though statistical significance was not reached, the cost savings resulting from pharmacists' recommendations to discontinue drugs that were no longer indicated or were duplicated, or to switch to cheaper alternatives, have a direct impact on patients and their families.

One limitation of the present study, however, is that only direct cost savings were calculated. Indirect cost savings, such as those resulting from reduced rates of hospitalisation and adverse outcomes, better management of undertreated or untreated conditions, and therapeutic monitoring for toxicity and effectiveness, were not addressed. We believe that if indirect cost savings were taken into account, the cost savings would have been considerably higher.

By quantifying the potential risks of the identified pharmacotherapy problems, we were able to observe the benefits of the pharmacist reviews, i.e. increasing medication safety and quality of care. There were 375 risks identified, and $16.0 \%$ of them were the risk of falls, which are potentially catastrophic 
for elderly patients. Constipation (13.1\%), a common and distressing adverse effect of some drugs, can also be prevented with pharmacist reviews. Other potentially hazardous risks such as hypoglycaemia, bleeding, drug allergy, poor seizure control or antiepileptic toxicity, hyperkalaemia and renal failure were also identified in the present study. For example, under a potentially hazardous risk identified, the pharmacist's notes described a patient who refused food and subsequently had a dangerously low blood glucose level of $1.3 \mathrm{mmol} / \mathrm{L}$ (Table VII); this low glucose level could cause the patient to fall into a coma or even lead to death. Therefore, it would be prudent to discontinue the patient's use of acarbose, as it would act on postprandial glucose if the patient was not eating well.

Another significant example involved the discontinuation of low-dose amlodipine in an 82-year-old patient with a fall history and blood pressure of $100 / 60 \mathrm{mmHg}$ to $120 / 90 \mathrm{mmHg}$ (Table VII). If the antihypertensive medication had not been discontinued, a fall episode would be inevitable and could result in other devastating effects such as fractures and head injuries. Apart from antihypertensive drugs, other potential culprits for falls are antihistamines, anticholinergics and benzodiazepines. However, it is important to note that some of these sedatives may be prescribed to prevent the elderly from wandering off alone, which can also be a fall hazard. Therefore, it is important for pharmacists to identify the best care management for the patient together with the multidisciplinary team. This can include pharmacological and non-pharmacological methods.

Prolonged NSAID exposure in elderly patients is potentially hazardous, as these drugs may carry significant dose-related risks of cardiovascular, renal, haematological and other side effects. ${ }^{(22)}$ Most NSAIDs can worsen chronic renal failure, particularly in patients with co-existing renal damage and patients who were prescribed diuretics or angiotensin-converting enzyme inhibitors. If NSAID therapy is prescribed in such patients, the lowest dose should be used for the shortest possible period and therapy should be reviewed regularly. ${ }^{(23)}$ The two cases of prolonged NSAID exposure identified by pharmacists (Table $\mathrm{V}$ ) in the present study proved that such reviews are helpful for ensuring that NSAIDS are discontinued if their use is unindicated or prolonged, thus improving medication safety and patient care.

Other than the aforementioned benefits, pharmacist reviews also enabled pharmacists to make important recommendations concerning potential pharmacotherapy problems. These include recommendations for the therapeutic monitoring of drugs with a narrow therapeutic window (e.g. antiepileptic drugs) and avoidance of drug toxicity in renal impaired patients (Table V). If the pharmacist's recommendations are ignored, the consequences of such potential pharmacotherapy problems can be dire. For example, inappropriate doses of antiepileptic drugs can result in uncontrolled seizures, which may lead to falls, while inappropriate doses of both antiepileptics and other drugs may lead to drug toxicity in renal patients. Therefore, pharmacist reviews act as a safety measure for nursing home residents and increases the quality of care in nursing homes. This benefit has also been demonstrated in a study conducted in nursing homes in Norway, where multidisciplinary meetings with pharmacists contributed to the critical evaluation of the quality of patients' drug treatment. ${ }^{(3)}$

The present study is not without limitations. First, the study is not a randomised trial; the nursing homes were selected because they had been set up within the time frame of the study. A randomised trial with a control arm consisting of nursing homes with similar demographics would produce more reliable data. However, although such a trial would be more highly regarded, it would face logistical restrictions, particularly manpower constraints. It would also be difficult to select control nursing homes, as variables other than demographics (e.g. nursing home practices, doctors' preferences) can confound the results. In view of these constraints, a before-after study design was used instead. Second, a direct comparison of cost savings between the pre- and post-setup periods was not done during the data analysis. This was because of the differences in manpower and duration between the two study phases (due to the unique pre- and post-setup methods employed by NHG Pharmacy). Despite this limitation, the analysis was able to show that pharmacist recommendations resulted in a huge difference in value between the two periods.

Third, indirect cost savings could not be measured in the present study, as we did not address the clinical outcomes (e.g. decreased rates of falls, hospitalisation and mortality) that resulted from the pharmacist reviews. The lack of a control arm also made it impossible to assess these clinical outcomes. Additionally, in order to analyse such rare outcomes, the sample size would have to be much larger. While we acknowledge that it would be more meaningful to compare these clinical outcomes, it was not within the capability of the present study. Finally, we are unable to comment on inter-rater reliability, as the data classification was performed by a single author. We acknowledge that this limits our ability to assess the consistency of the author's classification. However, open discussion among the authors was employed to reach a meaningful consensus for data analysis.

In conclusion, we recommend the provision of pharmaceutical care by pharmacists in nursing homes. As pharmacists are capable of identifying pharmacotherapy problems and recommending appropriate solutions to avert any risks, this would greatly improve the medication safety and quality of care for nursing home residents. Furthermore, in the present study, observable direct cost saving was noted during the post-setup period; although not statistically significant, there may also have been unquantifiable indirect cost savings (such as those derived from potential increase in quality of life and decrease in morbidity/mortality). Future research to determine both direct and indirect cost savings resulting from pharmacist reviews would be useful.

In order for pharmacist reviews to be successfully carried out in nursing homes, there is a need to build trust between the pharmacist and the multidisciplinary team, especially with physicians. This is because the physician-pharmacist relationship was historically characterised as conflictual and competitive. ${ }^{(20)}$ Halvorsen et al suggested that the pharmacist's expectations should be clarified with the multidisciplinary team members at an early stage. ${ }^{(6)}$ A trusting and collaborative relationship between the multidisciplinary team and the pharmacist can 
lead to better care management of nursing home residents. The post-setup model of pharmacist visits used in the present study, in which the pharmacist reviews the patients and/or participates in multidisciplinary case conferences weekly (as opposed to a longer interval), may help to improve this relationship. However, further studies to investigate the optimal interval for pharmacist reviews should be conducted.

\section{ACKNOWLEDGEMENTS}

We are grateful to Mr Stefan Kowalski, Programme Director of the Postgraduate Suite of Clinical Pharmacy Programmes at the University of South Australia, for vetting our manuscript and providing advice on the structure of the study write-up. We would also like to thank the pharmacists involved in the present study for their hard work reviewing the nursing home residents.

\section{REFERENCES}

1. Mamun K, Lien CT, Goh-Tan CY, Ang WS. Polypharmacy and inappropriate medication use in Singapore nursing homes. Ann Acad Med Singapore $2004 ; 33: 49-52$.

2. Stafford AC, Alswayan MS, Tenni PC. Inappropriate prescribing in older residents of Australian care homes. J Clin Pharm Ther 2011; 36:33-44.

3. Halvorsen KH, Ruths S, Granas AG, Viktil KK. Multidisciplinary intervention to identify and resolve drug-related problems in Norwegian nursing homes. Scand J Prim Health Care 2010; 28:82-8.

4. Forsetlund L, Eike MC, Gjerberg E, Vist GE. Effect of interventions to reduce potentially inappropriate use of drugs in nursing homes: a systematic review of randomised controlled trials. BMC Geriatr 2011; 11:16.

5. Tamura BK, Bell CL, Inaba M, Masaki KH. Outcomes of polypharmacy in nursing home residents. Clin Geriatr Med 2012; 28:217-36.

6. Halvorsen KH, Stensland P, Granas AG. A qualitative study of physicians' and nurses' experiences of multidisciplinary collaboration with pharmacists participating at case conferences. Int J Pharm Pract 2011; 19:350-7.

7. Ministry of Health, Singapore. Guidelines on medication management in nursing homes. Available at: https://elis.moh.gov.sg/elis/publishlnfo. do?task=download\&pkld=166. Accessed August 26, 2011.

8. Wagner $\mathrm{EH}$. The role of patient care teams in chronic disease management. BMJ 2000; 320:569-72.

9. Hoth $A B$, Carter BL, Ness J, et al. Development and reliability testing of the clinical pharmacist recommendation taxonomy. Pharmacotherapy 2007; 27:639-46.

10. Briesacher B, Limcangco R, Simoni-Wastila L, Doshi J, Gurwitz J. Evaluation of nationally mandated drug use reviews to improve patient safety in nursing homes: a natural experiment. J Am Geriatr Soc 2005; 53:991-6.

11. Tosato M, Landi F, Martone AM, et al. Potentially inappropriate drug use among hospitalised older adults: results from the CRIME study. Age Ageing 2014; 43:767-73.

12. American Geriatrics Society 2012 Beers Criteria Update Expert Panel. American Geriatrics Society updated Beers Criteria for potentially inappropriate medication use in older adults. J Am Geriatr Soc 2012; 60:616-31.

13. Gallagher P, O'Mahony D. STOPP (Screening Tool of Older Persons' potentially inappropriate Prescriptions): application to acutely ill elderly patients and comparison with Beers' criteria. Age and Ageing 2008; 37:673-9.

14. Vieira de Lima TJ, Garbin CA, Garbin AJ, Sumida DH, Saliba O. Potentially inappropriate medications used by the elderly: prevalence and risk factors in Brazilian care homes. BMC Geriatr 2013; 13:52.

15. Hepler CD, Strand LM. Opportunities and responsibilities in pharmaceutical care. Am J Hosp Pharm 1990; 47:533-43.

16. Hatah E, Tordoff J, Duffull SB, Braund R. Pharmacists' performance of clinical interventions during adherence support medication reviews. Res Social Adm Pharm 2014; 10:185-94.

17. Pharmaceutical Care Network Europe Foundation. Classification for Drug related problems V6.2. Available at: http://www.pcne.org/upload/ files/11_PCNE_classification_V6-2.pdf. Accessed April 7, 2014.

18. Alkhateeb FM, Unni E, Latif D, Shawaqfeh MS, Al-Rousan RM. Physician attitudes toward collaborative agreements with pharmacists and their expectations of community pharmacists' responsibilities in West Virginia. J Am Pharm Assoc (2003) 2009; 49:797-800.

19. Hatah E, Braund R, Duffull S, Tordoff J. General practitioners' perceptions of pharmacists' new services in New Zealand. Int J Clin Pharm 2012; 34:364-73.

20. Bradley F, Ashcroft DM, Noyce PR. Integration and differentiation: a conceptual model of general practitioner and community pharmacist collaboration. Res Social Adm Pharm 2012; 8:36-46.

21. Teinilä T, Kaunisvesi K, Airaksinen M. Primary care physicians' perceptions of medication errors and error prevention in cooperation with community pharmacists. Res Social Adm Pharm 2011; 7:162-79.

22. Barkin RL, Beckerman M, Blum SL, et al. Should nonsteroidal antiinflammatory drugs (NSAIDs) be prescribed to the older adult? Drugs Aging 2010; 27:775-89.

23. Abdulla A, Adams N, Bone M, et al. Guidance on the management of pain in older people. Age Ageing 2013; 42 Suppl 1:i1-57. 